\title{
Histiocytoid breast carcinoma, neither lobular nor ductal? A case report and literature review
}

\author{
Xiao-Li Ma ${ }^{1}$, Qian Du ${ }^{1}$, Yang Liu ${ }^{1}$, Rong-Mei Zhao ${ }^{2}$, Shu-Yan Zhang ${ }^{1}$, Huan-Fen Zhao ${ }^{1}$ \\ ${ }^{1}$ Department of Pathology, Hebei General Hospital, Shijiazhuang, China; ${ }^{2}$ Department of Ultrasonography, Hebei General Hospital, Shijiazhuang, \\ China \\ Correspondence to: Huan-Fen Zhao. Hebei General Hospital, NO. 348 of West He-Ping Road, Xin-Hua District, Shijiazhuang 050001, China. \\ Email: binglikezhaohf@163.com.
}

\begin{abstract}
Histiocytoid breast carcinoma (HBC) is a rare type of breast cancer with controversial histogenesis, which is characterized by abundant foamy cytoplasm, fuzzy cell boundary, linear or annular infiltration, eccentric large irregular nuclei or prominent nucleoli and low mitotic activity. HBC has been considered to be a variant of lobular carcinoma, a variant of apocrine ductal carcinoma, and an apocrine variant of lobular carcinoma and to resemble lipid-rich carcinoma. We presented a case of 75-year-old woman with a $5-\mathrm{cm}$ mass in the left breast. The mass was yellow-beige on cut section. HBC was diagnosed including invasive carcinoma (IC) of apocrine differentiation (diameter about $5 \mathrm{~mm}$ ) which was surrounded by extensive carcinoma in situ (CIS, diameter about $25 \mathrm{~mm}$ ) of apocrine type, and a 4-mm invasive ductal carcinoma (IDC) in grade II. The distance between HBC and IDC was $4 \mathrm{~mm}$. There was extensive (42 of 43 lymph nodes) metastasis and intravascular tumor emboli. The tumor extended into peripheral nerve. The pathology showed histiocytoid breast carcinoma with a smaller conventional invasive ductal carcinoma in adjacent area. She received a left modified radical mastectomy. However, on the follow-up imaging techniques, the mass showed no response. We discussed the pathology and immunohistochemical finding, and reviewed the literatures. We found that this case was a unique type of HBC.
\end{abstract}

Keywords: Case report; histiocytoid breast carcinoma (HBC); apocrine differentiation; invasive lobular carcinoma; invasive ductal carcinoma

Submitted Apr 12, 2019. Accepted for publication Aug 24, 2020.

doi: $10.21037 /$ tcr-19-756

View this article at: http://dx.doi.org/10.21037/tcr-19-756

\section{Introduction}

Histiocytoid breast carcinoma (HBC) is a rare tumor that was first described in 1973 by Hood et al. as lobular or ductal carcinoma. We reviewed the literatures and found that HBC was diagnosed as lobular carcinoma or ductal carcinoma by E-cadherin. In one case, the author added P120, but the E-cadherin and P120 were both negative. Our case exhibited the similar result. We suspected that the immunohistochemical indicators of HBC in the past may be not correct. Here, we reported a unique type of HBC. We present the following case in accordance with the CARE Reporting Checklist (available at http://dx.doi. org/10.21037/tcr-19-756).

\section{Case presentation}

A 75-year-old woman was found a mass in the left breast for 3 months. The mass showed unclear border with multiple lymph node swelling. Mammogram represented a poorly circumscribed mass in size of $5.0 \mathrm{~cm} \times 4.7 \mathrm{~cm} \times 3.0 \mathrm{~cm}$. Ultrasound images (Figure 1) displayed an inhomogeneous hypoechoic nodule in size of $29.1 \mathrm{~mm} \times 21.3 \mathrm{~mm}$. Ultrasound-guided fine needle aspiration biopsy revealed invasive ductal carcinoma. Thus, the patient underwent left modified radical mastectomy. Moreover, the patient has long-term history of antihypertensive medication. No family members have been diagnosed with breast cancer. Genetic-testing was omitted in clinical diagnosis. 

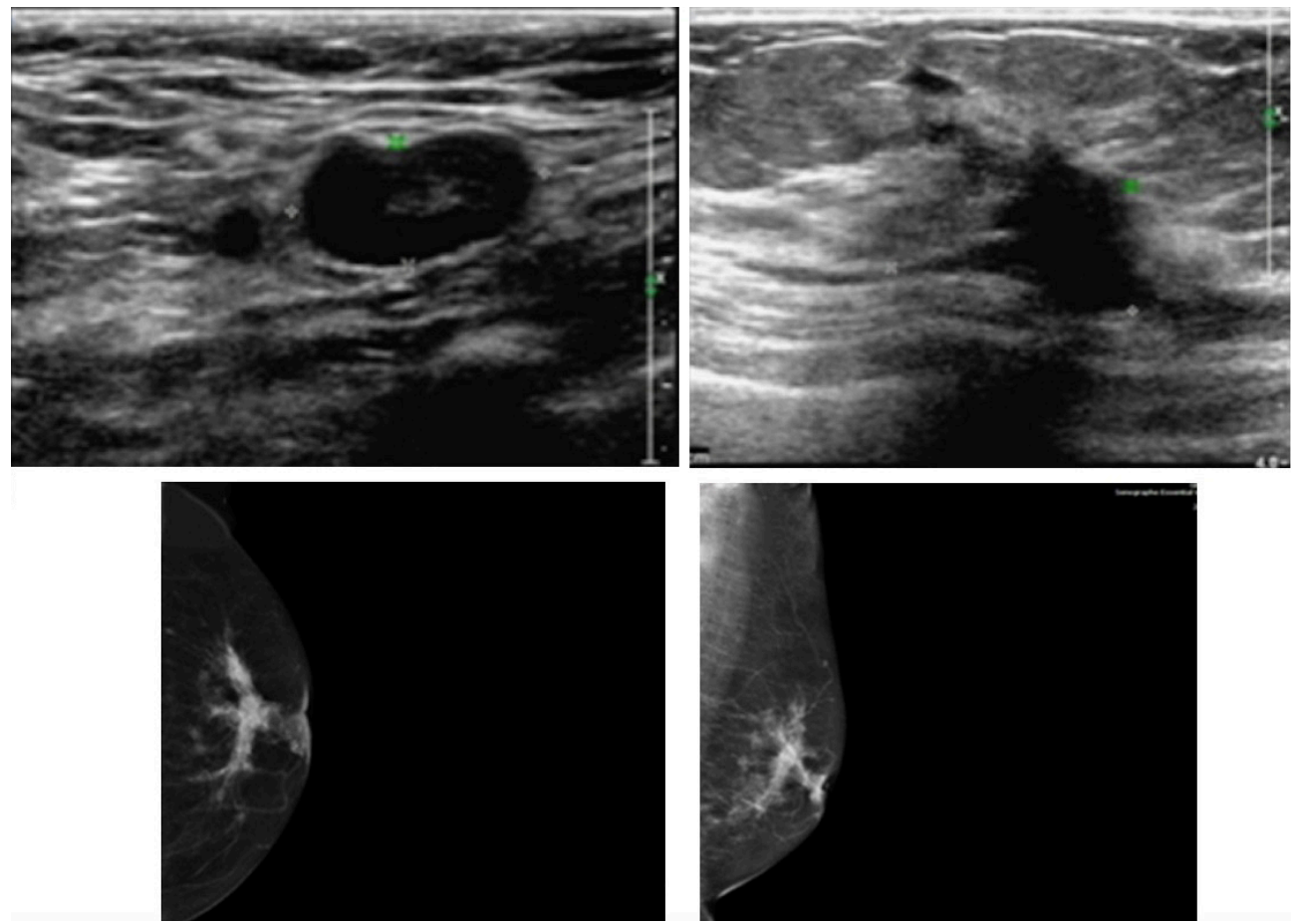

Figure 1 Ultrasound images. Ultrasound examination demonstrates a hypoechoic mass. Mammogram showed a poorly circumscribed mass.

Macroscopic pathological examination showed the tumor size as $3.0 \mathrm{~cm} \times 2.5 \mathrm{~cm} \times 2.0 \mathrm{~cm}$. The mass was yellowbeige on cut section. HBC was diagnosed including invasive carcinoma (IC) of apocrine differentiation (diameter about $5 \mathrm{~mm}$ ) which was surrounded by extensive carcinoma in situ (CIS, diameter about $25 \mathrm{~mm}$ ) of apocrine type, and a 4-mm invasive ductal carcinoma (IDC) in grade II. The distance between HBC and IDC was $4 \mathrm{~mm}$. There was extensive (42 of 43 lymph nodes) metastasis and intravascular tumor emboli. The tumor extended into peripheral nerve.

The tumor constituted by IDC and HBC which included IC and CIS. We compared the immunohistochemical profiles of 10 breast cancer markers among IC, CIS, and IDC (Table 1).

The invasive part of $\mathrm{HBC}$ varied from linear arrangements to irregular small clusters and isolated single cells. The tumor cells had abundant amphophilic cytoplasm, ranging from granular to vacuolated and feathery. They were characterized by enlarged discohesive cells with eccentric large irregular nuclei and prominent nucleoli. They displayed very low mitotic activity. The neoplastic cells demonstrated strong and diffuse immunoreactivity for gross cystic disease fluid protein (GCDFP-15) and AR which exhibited apocrine differentiation. However, both
Table 1 Immunohistochemical patterns of differentially marker proteins in HBC (including IC and CIS) and IDC

\begin{tabular}{lccc}
\hline Markers & IC (HBC) & CIS (HBC) & IDC \\
\hline GCDFP-15 & + & + & - \\
AR & + & + & - \\
E-cadherin & - & - & Membrane + \\
P120 & Cytoplasm- & Cytoplasm- & Membrane+ \\
CK5/6 & - & - & - \\
EGFR & - & - & - \\
ER & - & - & $90 \%,+++$ \\
PR & - & - & - \\
HER2 & $2+$ & $2+$ & $2+$ \\
P53 & + & + & - \\
\hline
\end{tabular}

-, negative. +, positive. IC: invasive carcinoma. CIS: carcinoma in situ. IDC: invasive ductal carcinoma.

membrane and cytoplasm were negative for E-cadherin and P120 staining. P120 was only non-specific staining due to cytoplasm richness. The diagnostic challenges are that apocrine carcinoma, lobular carcinoma, and 

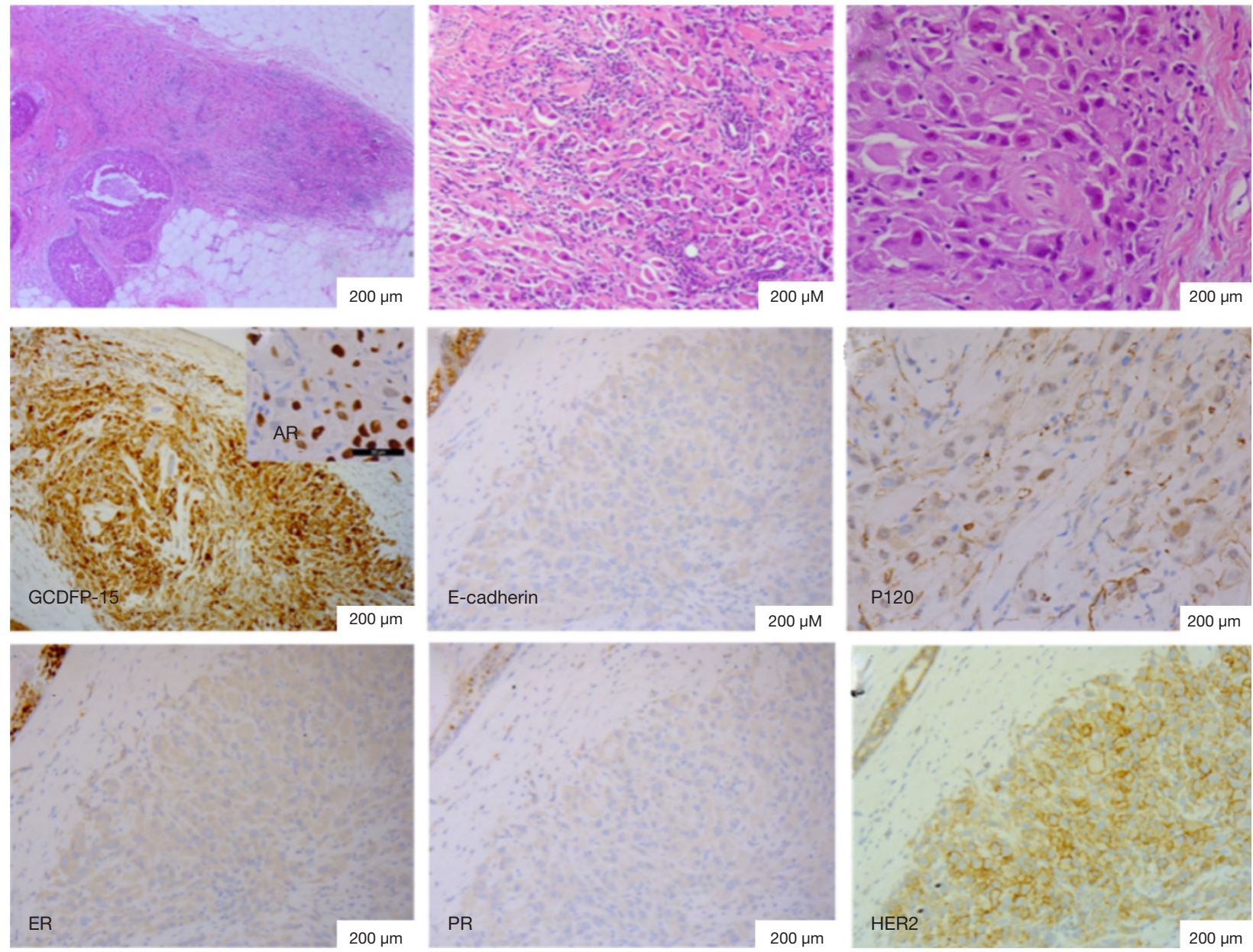

Figure 2 Microscopic findings. HBC included invasive carcinoma and carcinoma in situ. The cells contain abundant foamy or finely granular pale cytoplasm. Immunohistochemical findings: the IC of HBC was positive for GCDFP-15, AR and HER2; negative for E-cadherin, P120, ER and PR. H\&E Staining

ductal carcinoma were all excluded after sufficient immunohistochemistry. Therefore, the pathological type was considered as neither lobular nor ductal. HER2/neu was moderately positive (2+). Fluorescent in situ hybridazation (FISH) analysis of HER2 gene status was negative. The cells demonstrated no reactivity with ER, PR (Figure 2), CK5/6, or EGFR. On the other hand, $\mathrm{P} 53$ was positive.

The combined type near the HBC was IDC (4-mm) without apocrine feature. The neoplastic cells lacked GCDFP-15 and AR, but demonstrated strong and diffuse membrane expressions for E-cadherin and P120. The case was characterized by positive expression of ER, HER2, but lack of PR (Figure 3). Meanwhile, P53 was negative.

The CIS part of HBC also showed apocrine feature. It was showed that two type cells disclosed abundant foamy and granular eosinophilic cytoplasm. The immunohistochemical markers of CIS were same as IC (Figure 4).

Due to high lymph node metastasis rate, the postoperative course was uneventful and the patient was treated with capecitabine for $500 \mathrm{mg}$ twice daily. The patients could not tolerate hormonal therapy. Currently, the patient was disease free for 10 months after surgery. Then the follow-up diagnosis was breast cancer metastasis to liver and lung. The patient's family gave up further treatment, and the patient died 6 months later. The timeline of diagnosis and therapy was shown in Figure 5.

All procedures performed in studies involving human participants were in accordance with the ethical standards of the Ethics Committee of Hebei General Hospital 

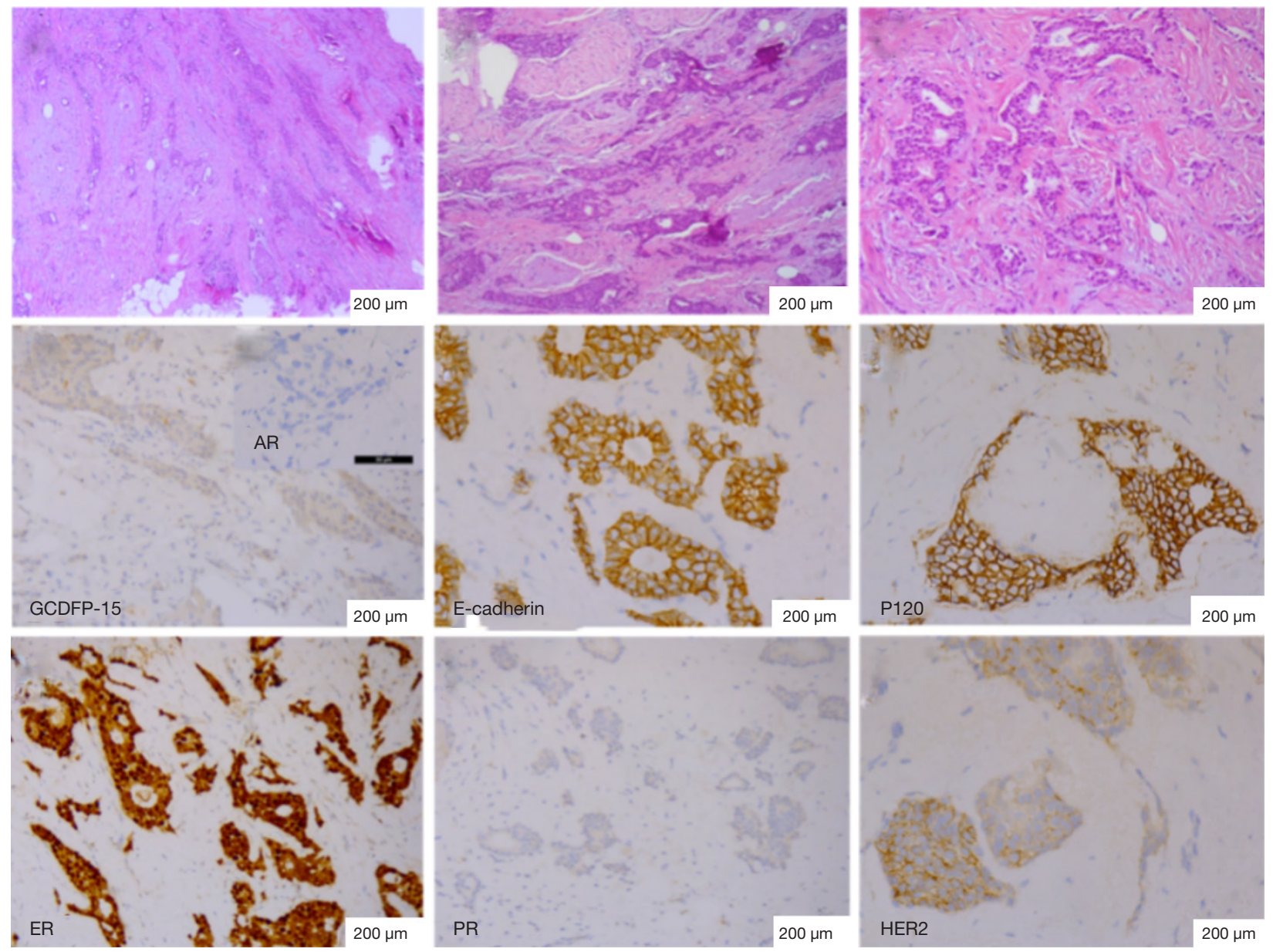

Figure 3 Microscopic findings. Invasive ductal carcinoma: the cells arranged in cords and clusters. Immunohistochemical findings: the cell was positive for E-cadherin, P120 (membrane+), ER and HER2; negative for GCDFP-15, AR and PR. H\&E Staining.

and with the Helsinki Declaration (as revised in 2013). Written informed consent was obtained from the patient for publication of this case report and any accompanying images.

\section{Discussion}

Despite being accepted as a variant of breast cancer by many pathologists, the term histio $\neg$ cytoid breast carcinoma (HBC) is yet to be included in the world health organization (WHO) classification of breast cancer due to its controversial histogenesis (1-3). It has been considered to be a variant of lobular carcinoma, a variant of apocrine ductal carcinoma, and an apocrine variant of lobular carcinoma and to resemble lipid-rich carcinoma (4-7). The majority of reports support the origin of $\mathrm{HBC}$ as lobular carcinoma and lobular carcinoma in situ. Some authors have concluded that $\mathrm{HBC}$ does not belong to a specific phenotype as both lobular and ductal origins are possible (8). E-cadherin and P120 are relatively specific immunohistochemical markers which were used for cell differentiation of breast carcinoma. However, our case presented the negative staining results.

In this case, the HBC part, whether invasive carcinoma or in situ, was triple negative. Contrastively, ER expression in the IDC part was positive. Based on triple negative phenotype, the HBC part is not that of classical lobular carcinoma as we know. Based on negative E-cadherin and $\mathrm{P} 120$, the HBC part is not lobular carcinoma and ductal carcinoma. Even pleomorphic lobular carcinoma, Her2 should be over expressed, while ER and PR should be positive. Gulbeyaz Omeroglu reported a case of triple negative histiocytoid variant of lobular carcinoma without 

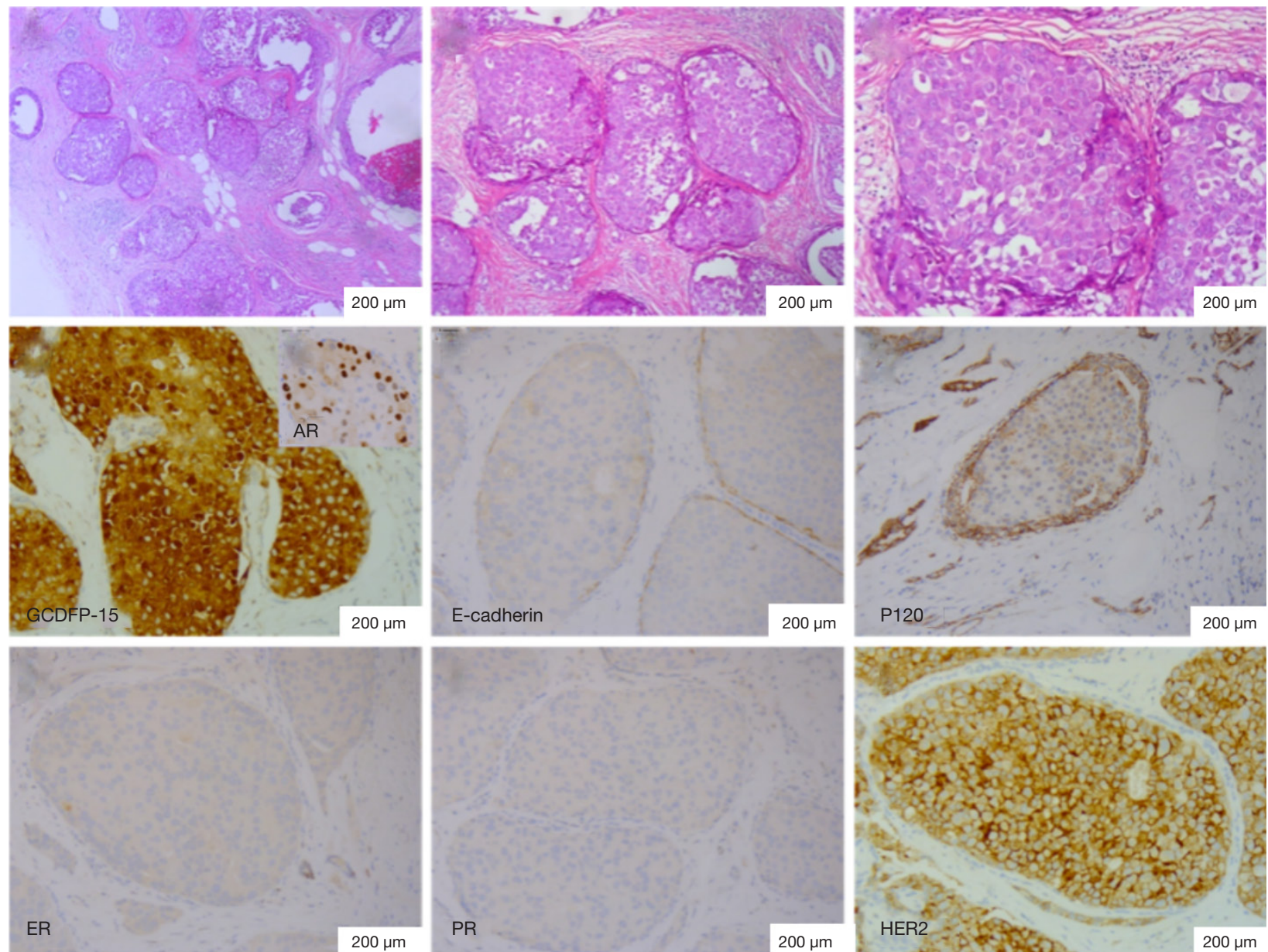

Figure 4 Microscopic findings. HBC (the part of CIS). Immunohistochemical findings: the cell was positive for GCDFP-15, AR and HER2; negative for E-cadherin, P120, ER and PR. H\&E Staining.

no operation history,

more than 10 years history of

antihypertensive medication

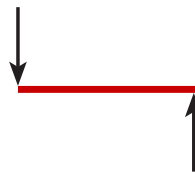

Surgical operation

on Oct $13^{\text {th }} 2015$

No received radiotherapy or

chemotherapy before operation
One day after chemotherapy,

patient intolerance and refused treatment

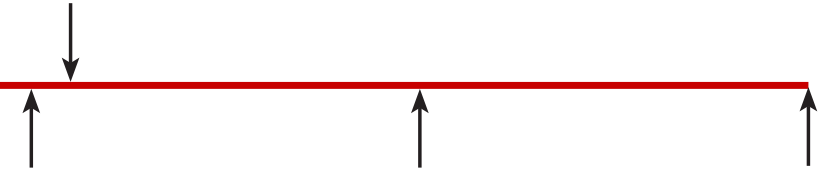

Two weeks after operation received epirubicin $\left(100 \mathrm{mg} / \mathrm{m}^{2}\right)$ combined with cyclophosphamide $\left(600 \mathrm{mg} / \mathrm{m}^{2}\right)$ treatment
Disease free for

10 months after surgery
Death on May $10^{\text {th }} 2019$

Figure 5 Timeline for the episode of care. 
E-cadherin and P120 expressions (9). Li et al. reported two HBC masses in one breast with negative expressions of E-cadherin and P120 (10). The author considered that it displayed characteristics more similar to lobular carcinoma than to ductal carcinoma with triple negative.

Gupta et al. described three E-cadherin positively stained cases and could therefore belong to ductal subtype. However, their E-cadherin positive case illustration showed just a few cells with apparent incomplete membrane staining, which may be not positive. The conclusion of ductal cancer may be not reliable. In not completely agreement with Eusebi et al., HBC could reasonably be defined as unique variant of breast cancer with histiocytoid cell (1). HBC is a unique variant of carcinoma originating in the terminal unit, without lobular and ductal differentiation.

Our case included a HBC and a ductal carcinoma occurring in one breast simultaneously. In the patient in our case report, the distance of two different types was $4 \mathrm{~mm}$. Their immunohistochemical phenotypes were completely different.

IHC studies classified breast carcinomas into four subtypes, including Luminal A, Luminal B, HER-2 over expression, and triple-negative breast cancer (TNBC) groups. Our case of HBC part was characterized by TNBC and lack expressions of CK5/6 and EGFR. This combination has been treated as a TNBC type. However, our case of mixed IDC part was characterized by ER expression and lack PR, HER2, CK5/6 and EGFR. Therefore, this combination was diagnosed as a Luminal A type. At last, the treatment after surgery was combined chemotherapy with endocrine.

The prognosis of patients with $\mathrm{HBC}$ was considered to be poor (11). More recent data, however, suggested that prognosis may depend on traditional prognostic factors (histological grade, tumor size, and lymph node status), as with more conventional breast carcinomas. More recent data, suggest that prognosis may depend on stage. In one reported case series, follow-up of 9 female patients demonstrated most patients alive with disease at 48 months, whereas this patient died at 42 months. The prognosis is not much different from that reported in the literature (12). On the other hand, this histiocytoid carcinomas are ER positive, which are favorable prognostic feature, but the patients could not tolerate hormonal therapy. Its consistent expression of GCDFP-15 revealed apocrine differentiation. The diagnosis of apocrine differentiation has not showed clinical significance up to now.

In this case report, we reported the first case of
HBC and IDC occurring in one breast. We concluded that histiocytoid breast carcinoma has an unconsistent immunophenotype with both ductal and lobular carcinomas. Moreover, the consistent morphologic features (histiocytoid cell feature) and a distinct immunohistochemical pattern induced us to hypothesize that histiocytoid breast carcinoma is a special type of breast cancer.

\section{Acknowledgments}

Funding: None.

\section{Footnote}

Reporting Checklist: The authors have completed the CARE reporting checklist. Available at http://dx.doi.org/10.21037/ tcr-19-756

Conflicts of Interest: All authors have completed the ICMJE uniform disclosure form (available at http://dx.doi. org/10.21037/tcr-19-756). The authors have no conflicts of interest to declare.

Ethical Statement: The authors are accountable for all aspects of the work in ensuring that questions related to the accuracy or integrity of any part of the work are appropriately investigated and resolved. All procedures performed in studies involving human participants were in accordance with the ethical standards of the Ethics Committee of Hebei General Hospital and with the Helsinki Declaration (as revised in 2013). Written informed consent was obtained from the patient for publication of this case report and any accompanying images.

Open Access Statement: This is an Open Access article distributed in accordance with the Creative Commons Attribution-NonCommercial-NoDerivs 4.0 International License (CC BY-NC-ND 4.0), which permits the noncommercial replication and distribution of the article with the strict proviso that no changes or edits are made and the original work is properly cited (including links to both the formal publication through the relevant DOI and the license). See: https://creativecommons.org/licenses/by-nc-nd/4.0/.

\section{References}

1. Eusebi V, Foschini MP, Bussolati G, et al. Myoblastomatoid (histiocytoid) carcinoma of the 
breast. A type of apocrine carcinoma. Am J Surg Pathol 1995;19:553-62.

2. Kostopoulos I, Barbanis S, Mylona E, et al. Histiocytoid breast carcinoma: a case report of an uncommon histologic variant of lobular carcinoma. Ann Pathol 2003;23:249-52.

3. Walford N, Ten VJ. Histiocytoid breast carcinoma: an apocrine variant of lobular carcinoma. Histopathology 1989;14:515-22.

4. Eisenberg BL, Bagnall JW, Harding CR. Histiocytoid carcinoma: a variant of breast cancer. J Surg Oncol 1986;31:271-4.

5. Gupta D, Croitoru CM, Ayala AG, et al. E-cadherin immunohistochemical analysis of histiocytoid carcinoma of the breast. Ann Diagn Pathol 2002;6:141-7.

6. Hood CI, Font RL, Zimmerman LE. Metastatic mammary carcinoma in the eyelid with histiocytoid appearance. Cancer 1973;31:793-800.

7. Winchester DJ, Chang HR, Graves TA, et al. A comparative analysis of lobular and ductal carcinoma of

Cite this article as: Ma XL, Du Q, Liu Y, Zhao RM, Zhang SY, Zhao HF. Histiocytoid breast carcinoma, neither lobular nor ductal? A case report and literature review. Transl Cancer Res 2020;9(11):7372-7378. doi: 10.21037/tcr-19-756 the breast: presentation, treatment, and outcomes. J Am Coll Surg 1998;186:416-22.

8. Filotico M, Trabucco M, Gallone D, et al. Histiocytoid carcinoma of the breast. A problem of differential diagnosis for the pathologist. Report of a case. Pathologica 1983;75:429-33.

9. Omeroglu G, Holloway CM, Spayne J, et al. Histiocytoid variant of lobular carcinoma: a triple negative case. Breast J 2010;16:84-6.

10. Li P, Zheng J, Zhang T, et al. Histiocytoid breast carcinoma: a case report showing immunohistochemical profiles. Int J Clin Exp Pathol 2013;6:2609-14.

11. Kasashima S, Kawashima A, Zen Y, et al. Expression of aberrant mucins in lobular carcinoma with histiocytoid feature of the breast. Virchows Arch 2007;450:397-403.

12. Gupta D, Croitoru C, Ayala A, et al. E-cadherin immunohistochemical analysis of histiocytoid carcinoma of the breast. Ann Diagn Pathol 2002;6:141-7. 